\title{
Highly Emissive Perylene Diimide-Based Metallacages and Their Host-Guest Chemistry for Information Encryption
}

Yali Hou, ${ }^{\dagger}$ Zeyuan Zhang, ${ }^{\dagger}$ Shuai Lu,,$\|$ Jun Yuan, ${ }^{\S}$ Qiangyu Zhu, ${ }^{\star}$ Wei-Peng Chen, ${ }^{\$}$ Sanliang Ling, ${ }^{\perp}$ Xiaopeng Li,,${ }^{\ddagger}$ Yan-Zhen Zheng, ${ }^{\$}$ Kelong Zhu, ${ }^{\S}$ Mingming Zhang, ${ }^{*}, \dagger$

†State Key Laboratory for Mechanical Behavior of Materials, Shaanxi International Research Center for Soft Matter, School of Materials Science and Engineering, Xi'an Jiaotong University, Xi'an 710049, P. R. China

$\$$ College of Chemistry and Environmental Engineering, Shenzhen University, Shenzhen 518055, P. R. China

$\|$ College of Chemistry and Molecular Engineering, Zhengzhou University, Zhengzhou 450001, P. R. China

$\S$ School of Chemistry, Sun Yat-Sen University, Guangzhou 510275, P. R. China

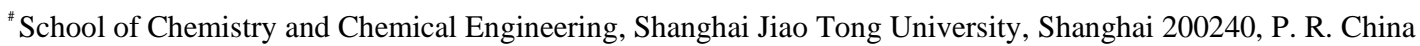

\$Frontier Institute of Science and Technology (FIST), Xi'an Jiaotong University, Xi'an 710049, P. R. China

${ }^{\perp}$ Advanced Materials Research Group, Faculty of Engineering, University of Nottingham, Nottingham, NG7 2RD, United Kingdom

Supporting Information Placeholder

ABSTRACT: Here we report two highly emissive perylene diimide (PDI)-based metallacages and explore their complexation with polycyclic aromatic hydrocarbons, such as pyrene, triphenylene and perylene. The fluorescence quantum yields of metallacages exceed $90 \%$ and their binding constants with perylene can reach as high as $2.41 \times 10^{4} \mathrm{M}^{-1}$ in acetonitrile. These features enable further tuning of the emission of the host-guest complexes to obtain white-light emission based on the complementary orange emission of the metallacages and the blue emission of perylene. Moreover, owing to the huge differences of their quantum yields in solution and in the solid state, the hostguest complexes are successfully employed for information encryption. This study offers a general approach for the construction of emissive metallacages and explores their application for information encryption.

Metal-organic cages or metallacages represent threedimensional supramolecular complexes formed by the coordination-driven self-assembly. ${ }^{1-10}$ Owing to their appealing structures and inner cavities that could be used for guest encapsulation, such metallacages have been widely used for absorption and separation, controlled release, catalysis, etc. ${ }^{11-16}$ The incorporation of fluorescent moieties into metallacages offers extra emissive functionalities, which enables their applications as chemical and biological sensors and light-emitting devices. ${ }^{17-19}$ However, because of the heavy-atom effects that often quench the emission after coordination and the metal-ligand charge transfer, highly emissive metallacages remain in their infancy. Stang and coworkers tackled this problem by introduction of tetraphenylethene (TPE) derivatives as the fluorophores to prepare a series of emissive metallacages. ${ }^{20-25}$ However, these metallacages are unable to bind organic guests, perhaps due to the twist configuration of TPE and the metallacycle-metallacage structural transition ${ }^{26-27}$ in solution. The exploration of the host-guest chemistry for emissive metallacages will not only offer a pathway to finely tune the emission of the complexes but also promote their use for photocatalytic reactions in confined spaces. Therefore, highly emissive metallacages with effective host-guest properties are urgently needed.

The introduction of efficient non-covalent interactions is a key issue to construct metallacages with effective host-guest chemistry. Since most metallacages are prepared using rigid organic ligands as their building blocks, the enhancement of $\pi-\pi$ stacking interactions seems to be the most convenient way to promote the host-guest interactions. Therefore, $\pi$-conjugated electron-deficient planar perylene diimide (PDI) and its derivatives are ideal building blocks for the preparation of metallacycles and metallacages ${ }^{28-}$ 32 that show good host-guest chemistry with electro-rich polycyclic aromatic hydrocarbons (PAHs). Moreover, their intense absorption, high fluorescence quantum yields $\left(\Phi_{\mathrm{F}}\right)$, good chemical and photo-stability will benefit the prepared metallacages with highly emissive properties.

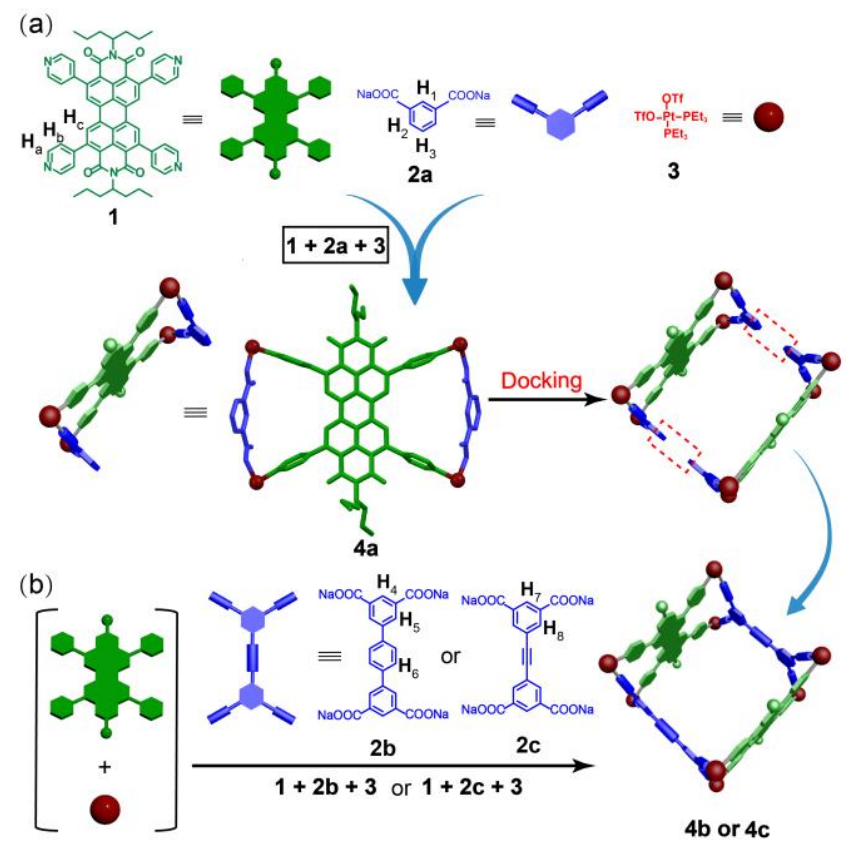

Scheme 1. (a) Self-assembly and crystal structure of doublemetallacycle $\mathbf{4 a}$, and (b) self-assembly of metallacages $4 \mathbf{b}$ and $\mathbf{4 c}$.

Herein, we report the preparation of two PDI-based metallacages via multicomponent self-assembly (Scheme 1) of a tetrapyridyl-PDI (1), tetracarboxylic ligands (2b or $\mathbf{2 c}$ ) and cis- $\mathrm{Pt}$ $\left(\mathrm{PEt}_{3}\right)_{2}(\mathrm{OTf})_{2}(\mathbf{3})$. Our design was inspired by the formation of double-metallacycle $\mathbf{4 a}$ via the self-assembly of $\mathbf{1}$, sodium isophthalate $2 \mathbf{a}$ and $\mathbf{3}$. In the crystal structure of $\mathbf{4 a}$, the two side benzene rings are parallel with each other. We speculated that the docking of two double-metallacycles in the benzene position would lead to the construction of metallacages. Therefore, two tetracarboxylic ligands $\mathbf{2 b}$ and $\mathbf{2 c}$ in which the two end benzene rings are parallel were employed for the self-assembly, resulting 
in highly emissive barrel-shaped metallacages $\mathbf{4 b}$ and $\mathbf{4 c}$ (Scheme 1), respectively. Moreover, these two metallacages can effectively encapsulate PAHs (pyrene, triphenylene, and perylene), offering a pathway to finely tune the emission of the host-guest systems. Interestingly, white-light emission was obtained in the complex $\mathbf{4 c} \supset$ perylene, which was used for selective information encryption owing to their different emissive properties in acetonitrile solution and in the solid state.

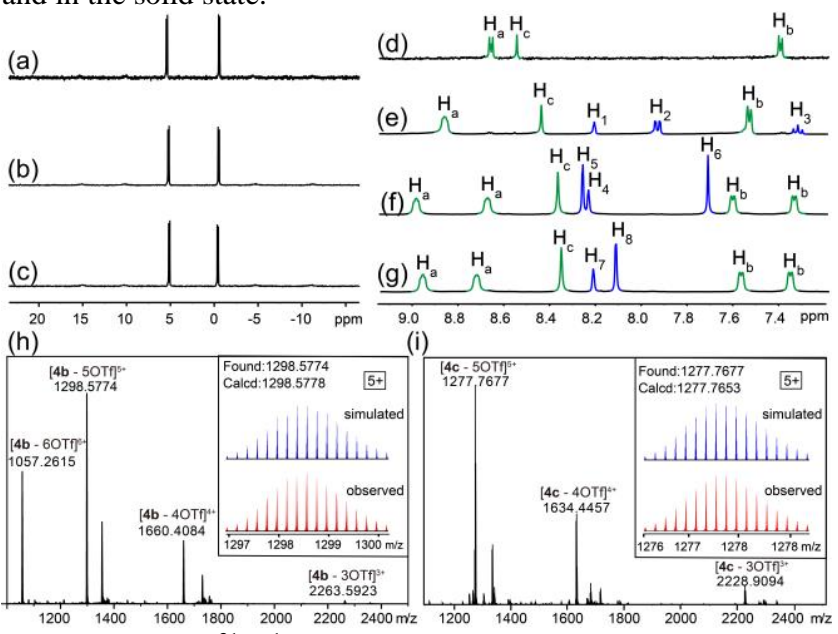

Figure 1. Partial ${ }^{31} \mathrm{P}\left\{{ }^{1} \mathrm{H}\right\}$ NMR spectra $\left(121.4 \mathrm{MHz}, \mathrm{CD}_{3} \mathrm{CN}, 295\right.$ $\mathrm{K}$ ) of (a) 4a, (b) $4 \mathrm{~b}$ and (c) 4c. Partial ${ }^{1} \mathrm{H}$ NMR spectra $(400 \mathrm{MHz}$, $\mathrm{CD}_{3} \mathrm{CN}, 295 \mathrm{~K}$ ) of (d) $\mathbf{1}$, (e) $\mathbf{4 a}$, (f) $\mathbf{4 b}$ and (g) $\mathbf{4 c}$. ESI-TOF-MS spectra of (h) $4 b$ and (i) $4 c$.

Multinuclear NMR $\left({ }^{31} \mathrm{P}\left\{{ }^{1} \mathrm{H}\right\}\right.$ and $\left.{ }^{1} \mathrm{H}\right)$ analysis and electrospray ionization time-of-flight mass spectrometry (ESI-TOF-MS) were carried out to support the formation of double-metallacycle $\mathbf{4 a}$ and metallacages $\mathbf{4 b}$ and $\mathbf{4 c}$. The ${ }^{31} \mathrm{P}\left\{{ }^{1} \mathrm{H}\right\}$ NMR spectra of $\mathbf{4 a}, \mathbf{4 b}$ and 4c split into two doublet peaks at $5.42 \mathrm{ppm}$ and $-0.56 \mathrm{ppm}$ for $\mathbf{4 a}$, $5.14 \mathrm{ppm}$ and $-0.41 \mathrm{ppm}$ for $\mathbf{4 b}$, and $5.22 \mathrm{ppm}$ and $-0.51 \mathrm{ppm}$ for 4c, (Figures 1a-c). These two doublet peaks share equal intensities with concomitant ${ }^{195} \mathrm{Pt}$ satellites owing to different phosphorus environments after the coordination of platinum atoms with pyridyl and carboxylic groups. In the ${ }^{1} \mathrm{H}$ NMR spectra (Figures 1d-g), compared to ligand 1, obvious downfield chemical shifts were found for $\alpha$-pyridyl protons $\mathrm{H}_{\mathrm{a}}$ and $\beta$-pyridyl protons $\mathrm{Hb}_{\mathrm{b}}$ of $\mathbf{4 a}, \mathbf{4 b}$ and $\mathbf{4 c}$ because of the decrease of electron densities induced by the coordination. It is worth noting that protons $\mathrm{H}_{\mathrm{a}}$ and $\mathrm{H}_{b}$ of metallacages $\mathbf{4 b}$ and $\mathbf{4 c}$ split into two sets of signals after coordination, corresponding to the protons inside and outside of the metallacages. ${ }^{1} \mathrm{H}$ DOSY spectra indicated that all the proton signals showed the same diffusion coefficient $(D)$ with $D=1.58 \times$ $10^{-9} \mathrm{~m}^{2} \mathrm{~s}^{-1}$ for $4 \mathbf{a}, D=6.88 \times 10^{-10} \mathrm{~m}^{2} \mathrm{~s}^{-1}$ for $4 \mathbf{b}$, and $D=7.63 \times 10^{-}$ $10 \mathrm{~m}^{2} \mathrm{~s}^{-1}$ for $4 \mathrm{c}$ in $\mathrm{CD}_{3} \mathrm{CN}$, suggesting the formation of single discrete structures. Based on the Stokes-Einstein equation, the hydrodynamic radius $\left(r_{\mathrm{s}}\right)$ of $\mathbf{4 a}, \mathbf{4 b}$ and $\mathbf{4 c}$ were calculated to be 0.41 $\mathrm{nm}, 0.94 \mathrm{~nm}$ and $0.85 \mathrm{~nm}$, respectively (Figure S16). ESI-TOFMS provided further evidence of the coordination stoichiometry of $\mathbf{4 b}$ and $\mathbf{4 c}$. Peaks at $\mathrm{m} / \mathrm{z}=1057.2615,1298.5774,1660.4084$, 2263.5923, 1277.7677, 1634.4457, 2228.9094 were found with isotopically well-resolved peaks, corresponding to $[4 \mathbf{b}-6 \mathrm{OTf}]^{6+}$, $[\mathbf{4 b}-5 \mathrm{OTf}]^{5+},[\mathbf{4 b}-4 \mathrm{OTf}]^{4+},[\mathbf{4 b}-3 \mathrm{OTf}]^{3+},[\mathbf{4} \mathbf{c}-5 \mathrm{OTf}]^{5+},[\mathbf{4 c}-$ $4 \mathrm{OTf}]^{4+}$ and $[\mathbf{4 c}-3 \mathrm{OTf}]^{3+}$, respectively.

Single crystals of $\mathbf{4 b}$ and $\mathbf{4 c}$ suitable for X-ray diffraction analysis were obtained by vapor diffusion of dioxane into DMF for three weeks. The crystal structures of $\mathbf{4 b}$ and $\mathbf{4 c}$ (Figures $2 \mathrm{a}$ and 2b) unambiguously confirmed the formation of three-dimensional metallacage structures. The eight $\mathrm{Pt}$ atoms behaved as a linker to connect the pyridyl and carboxylic groups, resulting in two barrelshaped metallacages $\mathbf{4 b}$ and $\mathbf{4 c}$. Different from previously report- ed TPE-based metallacage, ${ }^{20-25}$ these structures possess large windows that would allow guest molecules (e.g. PAHs) to enter into or out of the cavities. Using the distance between the platinum atoms, the length and width of metallacages $\mathbf{4 b}$ and $\mathbf{4 c}$ are 1.64 $\mathrm{nm} \times 1.44 \mathrm{~nm}$ and $1.64 \mathrm{~nm} \times 1.23 \mathrm{~nm}$, respectively. The metallacages are well-aligned to each other in their crystal structures, leading to the formation of one-dimensional channels (Figure S17) which may be useful for ion transport.

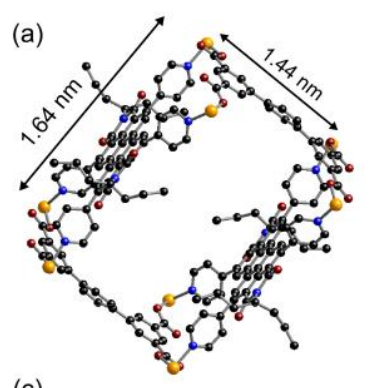

(c)

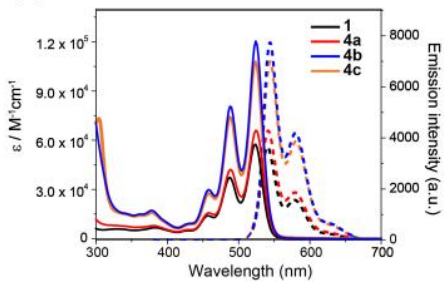

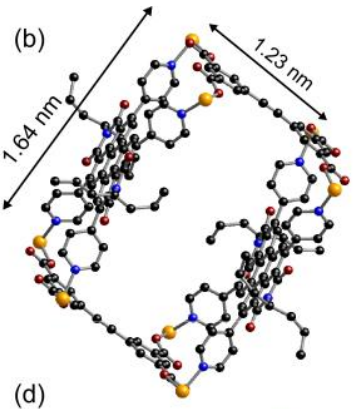

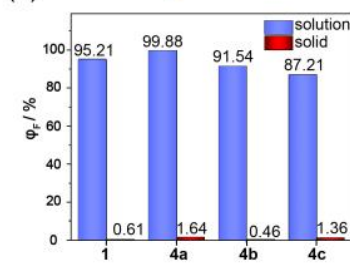

Figure 2. Crystal structures of (a) $\mathbf{4 b}$ and (b) 4c. Triethylphosphine units, hydrogen atoms, counterions and solvent molecules were omitted for clarity. (c) UV/Vis absorption (solid line) and emission (dash line) spectra of $\mathbf{1}, \mathbf{4 a}-\mathbf{c}$ in $\mathrm{CH}_{3} \mathrm{CN}$. (d) Fluorescence quantum yields of $\mathbf{1}, \mathbf{4 a - c}$ in $\mathrm{CH}_{3} \mathrm{CN}$ and in the solid state $\left(\lambda_{\mathrm{ex}}=365 \mathrm{~nm}, c=10.0 \mu \mathrm{M}, 295 \mathrm{~K}\right)$.

The UV/Vis absorption and emission spectra of ligand 1, double-metallacycle $\mathbf{4 a}$, metallacages $\mathbf{4 b}$ and $\mathbf{4 c}$ were collected (Figure 2c). Ligand 1 exhibits three absorption bands centered at 456 , 487 and $522 \mathrm{~nm}$ with molar absorption coefficients $(\varepsilon)$ of $1.40 \times$ $10^{4}, 3.68 \times 10^{4}$ and $5.74 \times 10^{4} \mathrm{M}^{-1} \mathrm{~cm}^{-1}$, respectively, consistent with the absorption of typical PDI derivatives. ${ }^{28,29}$ Metallacycle $\mathbf{4 a}$ and metallacages $\mathbf{4 b}$ and $\mathbf{4 c}$ show similar absorption bands with $\mathbf{1}$ owing to the weak absorption of the carboxylic ligands 2ac, with $\varepsilon=1.57 \times 10^{4}, 4.17 \times 10^{4}$ and $6.60 \times 10^{4} \mathrm{M}^{-1} \mathrm{~cm}^{-1}(\mathbf{4 a})$, $2.92 \times 10^{4}, 7.16 \times 10^{4}$ and $1.16 \times 10^{5} \mathrm{M}^{-1} \mathrm{~cm}^{-1}(4 \mathbf{b}), 1.57 \times 10^{4}$, $7.14 \times 10^{4}$ and $1.13 \times 10^{5} \mathrm{M}^{-1} \mathrm{~cm}^{-1}(\mathbf{4 c})$ at 456,488 and $524 \mathrm{~nm}$, respectively. Compared to ligand $\mathbf{1}$, the maximum emissions of metallacages $\mathbf{4 b}$ and $\mathbf{4 c}$ undergo a small red-shift (from $539 \mathrm{~nm}$ to $544 \mathrm{~nm}$ and from $576 \mathrm{~nm}$ to $578 \mathrm{~nm}$ ). Impressively, the fluorescence quantum yields $\left(\Phi_{\mathrm{F}}\right)$ of $\mathbf{4 a}, \mathbf{4 b}$ and $\mathbf{4} \mathbf{c}$ in $\mathrm{CH}_{3} \mathrm{CN}$ were measured to be $99.88 \%, 91.54 \%$, and $87.21 \%$, respectively. These values are among the highest quantum yields for supramolecular coordination complexes reported so far. ${ }^{17-21}$ However, the $\Phi_{\mathrm{F}}$ of the solid samples $\mathbf{4 a}, \mathbf{4 b}$ and $\mathbf{4 c}$ were $1.64 \%, 0.46 \%$, and $1.36 \%$, respectively, which are consistent with the values of PDI derivatives in the solid state (Figures S21-S28). ${ }^{28}$ Different from the TPE-based coordinated structures, ${ }^{20-25}$ these complexes display aggregation caused quenching (ACQ) behavior (Figure S39) ${ }^{33}$ due to the dense molecular packing which increases the non-radiative decay in the solid state.

Considering the barrel-like cavities and the electron-deficient properties of PDI derivatives, the complexation of metallacages $\mathbf{4 b}$ and $4 \mathbf{c}$ with electron-rich PAHs including pyrene $\left(\mathbf{G}_{\mathbf{1}}\right)$, triphenylene $\left(\mathbf{G}_{\mathbf{2}}\right)$, and perylene $\left(\mathbf{G}_{\mathbf{3}}\right)$ was further studied. Job's plots based on UV/Vis spectroscopic absorbance data $(\lambda=488 \mathrm{~nm})$ demonstrated that the complexes of $\mathbf{4 b}$ and $\mathbf{4 c}$ with $\mathbf{G}_{\mathbf{1}}, \mathbf{G}_{\mathbf{2}}$ and $\mathbf{G}_{\mathbf{3}}$ 
in solution were all of 1:1 stoichiometry (Figures S40-S45). This was also confirmed by ESI-TOF-MS spectra (Figures S46-S51). Taking complex $\mathbf{4} \mathbf{c} \supset \mathbf{G}_{3}$ as an example, peaks were found at $\mathrm{m} / \mathrm{z}=$ 1328.1190, 1697.4087 and 2312.9080, corresponding to [4c $\supset \mathbf{G}_{3}-$ 5OTf $]^{5+},[\mathbf{4} \mathbf{c} \supset \mathbf{G} 3-4 \mathrm{OTf}]^{4+}$ and $[\mathbf{4} \mathbf{c} \supset \mathbf{G} \mathbf{3}-3 \mathrm{OTf}]^{3+}$, respectively (Figure S51). The complexation between the metallacages $\mathbf{4 b}$ and 4c with guests were further studied by ${ }^{1} \mathrm{H}$ NMR spectroscopy (Figure 3). All the complexation systems are fast-exchange on the proton NMR timescale, because the large windows of the metallacages facilitate the guests to get in and out. Significant upfield shifts were observed for all the aromatic protons on the guests, indicating the good host-guest interactions. The binding constants $\left(K_{\mathrm{a}}\right)$ of the host-guest complexes were determined by ${ }^{1} \mathrm{H} \mathrm{NMR}$ titration experiments in $\mathrm{CD}_{3} \mathrm{CN}$ (Figures S52-S57) and the data were listed in Figure 3. These values are among the highest binding constants for emissive metallacage-based host-guest interactions. ${ }^{20-32}$ The binding affinity of metallacages towards PAHs is attributed to the $\pi-\pi$ stacking interactions between the electrondeficient PDI and the electron-rich PAHs. Because of slightly different sizes and shapes of the three PAHs, it is likely that they will have different binding modes and energies with PDI-based metallacages, which gives them different binding affinities with the two metallacages.

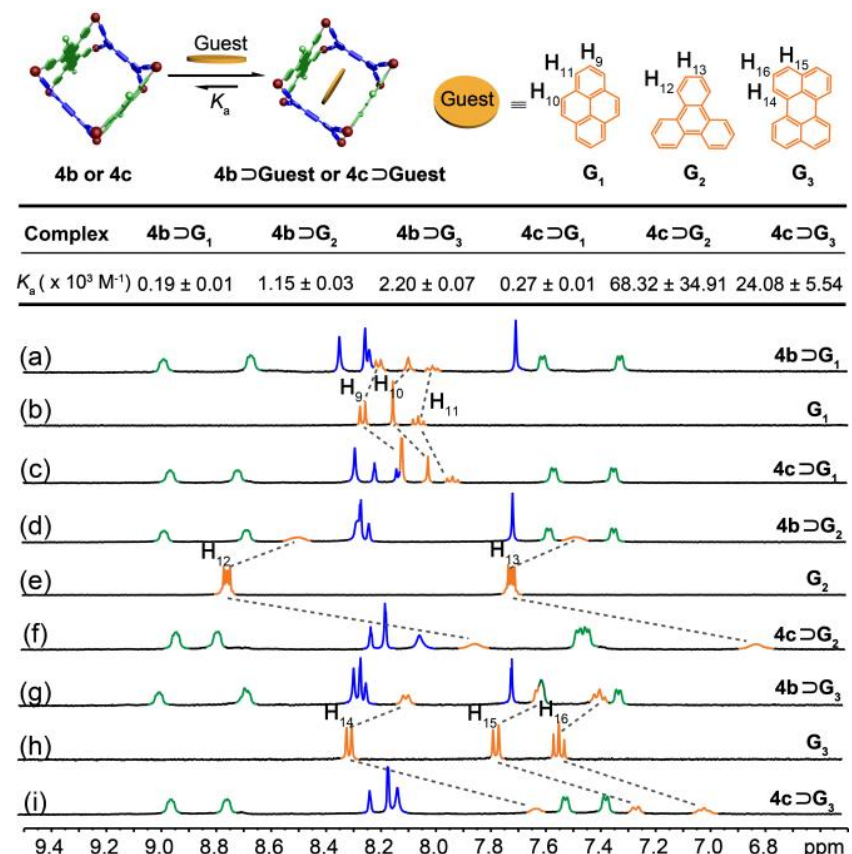

Figure 3. Cartoon representations of the complexation between metallacages and PAHs and their binding constants. Partial ${ }^{1} \mathrm{H}$ NMR spectra (400 MHz, CD 3 CN, $295 \mathrm{~K}$ ) of (a) $\mathbf{4 b} \supset \mathbf{G}_{\mathbf{1}}$, (b) $\mathbf{G} \mathbf{1}$,

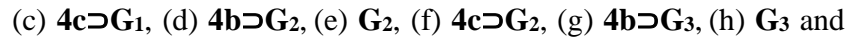
(i) $\mathbf{4} \mathbf{c} \supset \mathbf{G}_{3}$. $[$ Host $]=[$ Guest $]=2.00 \mathrm{mM}$.

The emission of metallacage $\mathbf{4 c}$ with increasing amount of perylene $\mathbf{G}_{3}$ in $\mathrm{CH}_{3} \mathrm{CN}$ was investigated by fluorescence spectroscopy. As seen in Figure 4a, the addition of different amounts of $\mathbf{G}_{3}$ into the acetonitrile solution of $\mathbf{4 c}$ leads to the increase of blue emission derived from perylene, with the emission color of the solution changes from orange to blue (Figure 4c). Interestingly, because metallacage $\mathbf{4 c}$ shows orange emission and perylene emits in the blue region, white-light emission (CIE chromaticity coordinate: $0.29,0.33$ ) was readily achieved when 1.0 equivalent of perylene was added (Figure $4 \mathrm{~b}$ ). The host-guest complex $\mathbf{4 b} \supset \mathbf{G}_{\mathbf{3}}$ also shows similar behavior (Figure S58). This feature makes the metallacage-based host-guest systems serve as smart light-emitting materials.

(a)
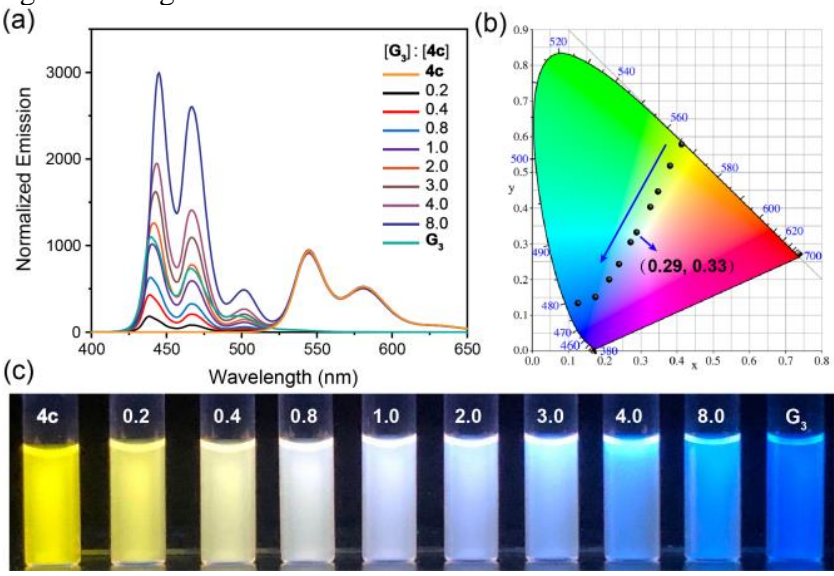

Figure 4. (a) Emission spectra of metallacage $\mathbf{4 c}$ with increasing amount of $\mathbf{G}_{3}$, (b) their corresponding 1931 CIE chromaticity coordinates and (c) fluorescence photographs in $\mathrm{CH}_{3} \mathrm{CN}$. ([4c] = $\left.10 \mu \mathrm{M}, \lambda_{\mathrm{ex}}=365 \mathrm{~nm}, 295 \mathrm{~K}\right)$.

Considering the distinct emissive feature of these metallacages and their host-guest complexes in solution and in the solid state, a colorful lantern pattern was printed on a paper using $\mathbf{G} 3, \mathbf{4} \mathbf{c}$ and $\mathbf{4} \supset_{\mathbf{G}}$ ( $\left.\left[\mathbf{G}_{3}\right]:[\mathbf{4}]=1: 1\right)$ as inks. The inks were loaded separately into a tricolor inkjet cartridge to separate the colors on paper. Interestingly, the lantern pattern was dim under natural light and UV light, but turned out to be quite bright immediately when it was sprayed with $\mathrm{CH}_{3} \mathrm{CN}$ vapor. Especially, the area printed by $\mathbf{4} \mathbf{c} \supset \mathbf{G}_{3}$ became white. This indicated that the host-guest complexes could be used for information encryption. A sentence ("Be emotional, you'll own nice days") was inkjet-printed on a nonfluorescent paper, with only selective letters ("Beyond") were printed using $\mathbf{4} \mathbf{c} \supset \mathbf{G}_{\mathbf{3}}$ as the ink. In the encrypted state, all the letters showed the same colors upon UV irradiation (Figure 5b, Movie 1) because $\mathbf{4} \mathbf{c} \supset \mathbf{G} 3$ is nearly non-emissive in the solid state $\left(\Phi_{\mathrm{F}}=0.16 \%\right)$. When the letters were exposed to $\mathrm{CH}_{3} \mathrm{CN}$ vapor, the word "Beyond" was turned on owing to the highly emissive nature of $\mathbf{4} \mathbf{c} \supset \mathbf{G}_{3}$ in $\mathrm{CH}_{3} \mathrm{CN}\left(\Phi_{\mathrm{F}}=94.97 \%\right)$. Upon drying, the information was encrypted again. This process is fully reversible, offering a convenient method for selective information encryption. (a)

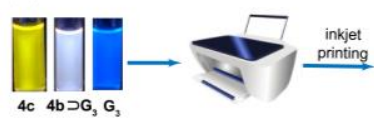

(b)
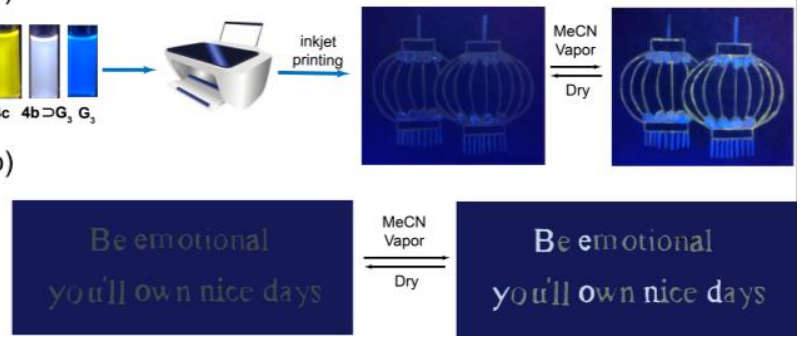

Figure 5. (a) Representations of colorful lantern pattern printed by three different inks and (b) information encryption using $\mathbf{4} \boldsymbol{c} \supset \mathbf{G}_{3}$ as the ink $\left(\lambda_{\mathrm{ex}}=365 \mathrm{~nm}\right)$.

In summary, two highly emissive metallacages which exhibited well-controlled host-guest interactions with PAHs were prepared. This feature provides a convenient pathway to finely tune the emission of the host-guest complexes, in which white-light emission was obtained on account of the complementary emission colors of the metallacages and perylene. The host-guest systems were further employed for selective information encryption. This study not only constructs a type of highly emissive metallacages as supramolecular hosts, but also explores their applications for 
information encryption, which will open an avenue for the applications of emissive metallacages as smart and adaptive materials.

\section{ASSOCIATED CONTENT}

\section{Supporting Information}

Experimental details and additional data. This material is available free of charge via the Internet at http://pubs.acs.org.

\section{AUTHOR INFORMATION}

\section{Corresponding Author}

*mingming.zhang@xjtu.edu.cn

\section{Notes}

The authors declare no competing financial interests.

\section{ACKNOWLEDGMENT}

This work was supported by the National Natural Science Foundation of China (21801203), The Key Research and Development Program of Shaanxi Province (2019KW-019) and Basic Research Program of Xi'an Jiaotong University (XZY022020018). We thank Dr. Gang Chang and Yu Wang at Instrument Analysis Center and Dr. Aqun Zheng and Junjie Zhang at Experimental Chemistry Center of Xi'an Jiaotong University for NMR and fluorescence measurements. We thank Dr. Li-Xuan Cai for the measurements of X-ray crystal structures. We also acknowledge the mass spectrometry characterization by Molecule Scale Lab.

\section{REFERENCES}

(1) Inokuma, Y.; Kawano, M.; Fujita, M. Crystalline Molecular Flasks. Nat. Chem. 2011, 3, 349-358.

(2) Brown, C. J.; Toste, F. D.; Bergman, R. G.; Raymond, K. N. Supramolecular Catalysis in Metal-Ligand Cluster Hosts. Chem. Rev. 2015, 115, 3012-3035.

(3) Cook, T. R.; Stang, P. J. Recent Developments in the Preparation and Chemistry of Metallacycles and Metallacages via Coordination. Chem. Rev. 2015, 115, 7001-7045.

(4) Clever, G. H.; Punt, P. Cation-Anion Arrangement Patterns in Self-Assembled $\mathrm{Pd}_{2} \mathrm{~L}_{4}$ and $\mathrm{Pd}_{4} \mathrm{~L}_{8}$ Coordination Cages. Acc. Chem. Res. 2017, 50, 2233-2243.

(5) Newkome, G. R. Terpyridine-Based Metallosupramolecular Constructs: Tailored Monomers to Precise 2D-Motifs and 3DMetallocages. Chem. Soc. Rev. 2018, 47, 3991-4016.

(6) Rizzuto, F. J.; von Krbek, L. K. S.; Nitschke, J. R. Strategies for Binding Multiple Guests in Metal-Organic Cages Nat. Rev. Chem. 2019, 3, 204-222.

(7) Yoshizawa, M.; Catti, L. Bent Anthracene Dimers as Versatile Building Blocks for Supramolecular Capsules. Acc. Chem. Res. 2019, 52, 2392-2404.

(8) Pan, M.; Wu, K.; Zhang, J.-H.; Su, C.-Y. Chiral Metal-Organic Cages/Containers (MOCs): From Structural and Stereochemical Design to Applications. Coord. Chem. Rev. 2019, 378, 333-349.

(9) Sun, Y.; Chen, C.; Liu, J.; Stang, P. J. Recent Developments in the Construction and Applications of Platinum-Based Metallacycles and Metallacages via Coordination. Chem. Soc. Rev. 2020, 49, 38893919.

(10) Jin, Y.; Zhang, Q.; Zhang, Y.; Duan, C. Electron transfer in the Confined Environments of Metal-Organic Coordination Supramolecular Systems. Chem. Soc. Rev. 2020, 49, 5561-5600.
(11) Kishi, N.; Li, Z.; Yoza, K.; Akita, M.; Yoshizawa, M. An $\mathrm{M}_{2} \mathrm{~L}_{4}$ Molecular Capsule with an Anthracene Shell: Encapsulation of Large Guests up to $1 \mathrm{~nm}$. J. Am. Chem. Soc. 2011, 133, 11438-11441.

(12) Garcia-Simon, C.; Garcia-Borras, M.; Gomez, L.; Parella, T.; Osuna, S.; Juanhuix, J.; Imaz, I.; Maspoch, D.; Costas, M.; Ribas, X. Sponge-Like Molecular Cage for Purification of Fullerenes. Nat. Commun. 2014, 5, 5557.

(13) Brenner, W.; Ronson, T. K.; Nitschke, J. R. Separation and Selective Formation of Fullerene Adducts within an $\mathrm{M}^{\mathrm{II}}{ }_{8} \mathrm{~L}_{6}$ Cage. $J$. Am. Chem. Soc. 2017, 139, 75-78.

(14) Cai, L. X.; Li, S. C.; Yan, D. N.; Zhou, L. P.; Guo, F.; Sun, Q. F. A Water-Soluble Redox-Active Cage Hosting Polyoxometalates for Selective Desulfurization Catalysis. J. Am. Chem. Soc. 2018, 140, 4869-4876.

(15) Takezawa, H.; Shitozawa, K.; Fujita, M. Enhanced Reactivity of Twisted Amides Inside a Molecular Cage. Nat. Chem. 2020, 12 , 574-578.

(16) Wu, K.; Li, K.; Chen, S.; Hou, Y. J.; Lu, Y. L.; Wang, J. S.; Wei, M. J.; Pan, M.; Su, C. Y. The Redox Coupling Effect in a Photocatalytic Ru(II)-Pd(II) Cage with TTF Guest as Electron Relay Mediator for Visible-Light Hydrogen-Evolving Promotion. Angew. Chem. Int. Ed. 2020, 59, 2639-2643.

(17) Neelakandan, P. P.; Jimenez, A.; Nitschke, J. R. Fluorophore Incorporation Allows Nanomolar Guest Sensing and White-Light Emission in $\mathrm{M}_{4} \mathrm{~L}_{6}$ Cage Complexes. Chem. Sci. 2014, 5, 908-915.

(18) Yamashina, M.; Sartin, M. M.; Sei, Y.; Akita, M.; Takeuchi, S.; Tahara, T.; Yoshizawa, M. Preparation of Highly Fluorescent HostGuest Complexes with Tunable Color upon Encapsulation. J. Am. Chem. Soc. 2015, 137, 9266-9269.

(19) Jing, X.; He, C.; Yang, Y.; Duan, C. A Metal-Organic Tetrahedron as a Redox Vehicle to Encapsulate Organic Dyes for Photocatalytic Proton Reduction. J. Am. Chem. Soc. 2015, 137, 39673974.

(20) Yan, X.; Cook, T. R.; Wang, P.; Huang, F.; Stang, P. J. Highly Emissive Platinum(II) Metallacages. Nat. Chem. 2015, 7, 342-348.

(21) Zhang, M.; Saha, M. L.; Wang, M.; Zhou, Z.; Song, B.; Lu, C.; Yan, X.; Li, X.; Huang, F.; Yin, S.; Stang, P. J. Multicomponent Platinum(II) Cages with Tunable Emission and Amino Acid Sensing. J. Am. Chem. Soc. 2017, 139, 5067-5074.

(22) Lu, C.; Zhang, M.; Tang, D.; Yan, X.; Zhang, Z.; Zhou, Z.; Song, B.; Wang, H.; Li, X.; Yin, S.; Sepehrpour, H.; Stang, P. J. Fluorescent Metallacage-Core Supramolecular Polymer Gel Formed by Orthogonal Metal Coordination and Host-Guest Interactions. J. Am Chem. Soc. 2018, 140, 7674-7680.

(23) Zhang, Z.; Zhao, Z.; Hou, Y.; Wang, H.; Li, X.; He, G.; Zhang, M. Aqueous Platinum(II) Cage-Based Light-Harvesting System for Photocatalytic Cross-Coupling Hydrogen Evolution Reaction. Angew. Chem. Int. Ed. 2019, 58, 8862-8866.

(24) Zhang, Z.; Zhao, Z.; Wu, L.; Lu, S.; Ling, S.; Li, G.; Xu, L.; Ma, L.; Hou, Y.; Wang, X.; Li, X.; He, G.; Wang, K.; Zou, B.; Zhang, M. Emissive Platinum(II) Cages with Reverse Fluorescence Resonance Energy Transfer for Multiple Sensing. J. Am. Chem. Soc. 2020, 142, 2592-2600.

(25) Li, G.; Zhou, Z.; Yuan, C.; Guo, Z.; Liu, Y.; Zhao, D.; Liu, K. Zhao, J.; Tan, H.; Yan, X. Trackable Supramolecular Fusion: Cage to Cage Transformation of Tetraphenylethylene-Based Metalloassemblies. Angew. Chem. Int. Ed. 2020, 59, 10013-10017.

(26) Li, C.; Nian, H.; Dong, Y.; Li, Y.; Zhang, B.; Cao, L. Tetraphenylethene-Based Platinum(II) Bis-Triangular Dicycles with Tunable Emissions. Inorg. Chem. 2020, 59, 5713-5720.

(27) Yang, Z.; Wang, Y.; Liu, X.; Vanderlinden, R. T.; Ni, R.; Li, X.; Stang, P. J. Hierarchical Self-Assembly of a Pyrene-Based Discrete Organoplatinum(II) Double-Metallacycle with Triflate Anions via Hydrogen Bonding and Its Tunable Fluorescence Emission. J. Am. Chem. Soc. 2020, 142, 13689-13694.

(28) Würthner, F.; Saha-Möller, C. R.; Fimmel, B.; Ogi, S.; Leowanawat, P.; Schmidt, D. Perylene Bisimide Dye Assemblies as Archetype Functional Supramolecular Materials. Chem. Rev. 2015, 116, 962-1052. 
(29) Chen, S.; Slattum, P.; Wang, C.; Zang, L. Self-Assembly of Perylene Imide Molecules into 1D Nanostructures: Methods, Morphologies, and Applications. Chem. Rev. 2015, 115, 11967-11998.

(30) Mahata, K.; Frischmann, P. D.; Würthner, F. Giant Electroactive $\mathrm{M}_{4} \mathrm{~L}_{6}$ Tetrahedral Host Self-Assembled with $\mathrm{Fe}(\mathrm{II})$ Vertices and Perylene Bisimide Dye Edges. J. Am. Chem. Soc. 2013, 135, 15656-15661.

(31) Frischmann, P. D.; Kunz, V.; Würthner, F. Bright Fluorescence and Host-Guest Sensing with a Nanoscale $\mathrm{M}_{4} \mathrm{~L}_{6}$ Tetrahedron Accessed by Self-Assembly of Zinc-Imine Chelate Vertices and Perylene Bisimide Edges. Angew. Chem. Int. Ed. 2015, 54, 7285-7289.

(32) Chang, X.; Lin, S.; Wang, G.; Shang, C.; Wang, Z.; Liu, K.; Fang, Y.; Stang, P. J. Self-Assembled Perylene Bisimide-Cored Trigonal Prism as an Electron-Deficient Host for $\mathrm{C}_{60}$ and $\mathrm{C}_{70}$ Driven by 'Like Dissolves Like'. J. Am. Chem. Soc. 2020, 142, 15950-15960. (33) Mei, J.; Leung, N. L.; Kwok, R. T.; Lam, J. W.; Tang, B. Z. Aggregation-Induced Emission: Together We Shine, United We Soar! Chem. Rev. 2015, 115, 11718-11940.

\section{TOC Graphic:}

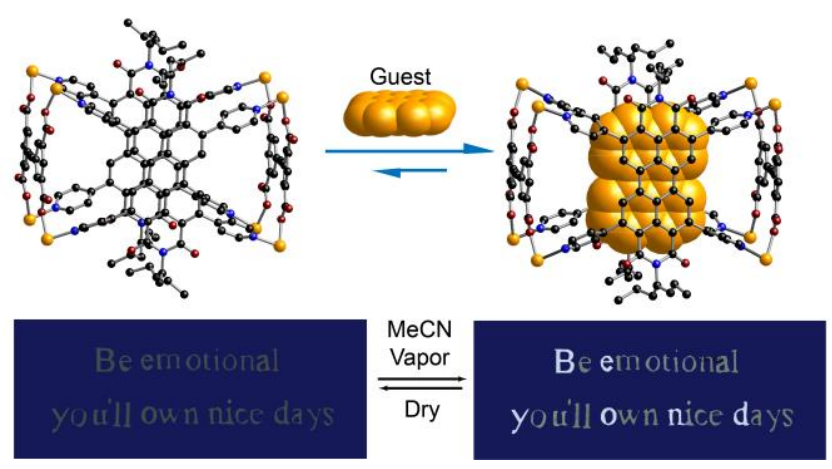

